\title{
Review Article \\ Endoscopic Raman Spectroscopy for Molecular Fingerprinting of Gastric Cancer: Principle to Implementation
}

\author{
Hyung Hun Kim \\ Department of Internal Medicine, College of Medicine, The Catholic University of Korea, Seoul, Republic of Korea \\ Correspondence should be addressed to Hyung Hun Kim; drhhkim@gmail.com
}

Received 7 December 2014; Revised 25 March 2015; Accepted 30 March 2015

Academic Editor: Michael Idowu

Copyright (C) 2015 Hyung Hun Kim. This is an open access article distributed under the Creative Commons Attribution License, which permits unrestricted use, distribution, and reproduction in any medium, provided the original work is properly cited.

\begin{abstract}
Currently, positive endoscopic biopsy is the standard criterion for gastric cancer diagnosis but is invasive, often inconsistent, and delayed although early detection and early treatment is the most important policy. Raman spectroscopy is a spectroscopic technique based on inelastic scattering of monochromatic light. Raman spectrum represents molecular composition of the interrogated volume providing a direct molecular fingerprint. Several investigations revealed that Raman spectroscopy can differentiate normal, dysplastic, and adenocarcinoma gastric tissue with high sensitivity and specificity. Moreover, this technique can indentify malignant ulcer and showed the capability to analyze the carcinogenesis process. Automated on-line Raman spectral diagnostic system raised possibility to use Raman spectroscopy in clinical field. Raman spectroscopy can be applied in many fields such as guiding a target biopsy, optical biopsy in bleeding prone situation, and delineating the margin of the lesion. With wide field technology, Raman spectroscopy is expected to have specific role in our future clinical field.
\end{abstract}

\section{Introduction}

In the era of minimally destructive treatment, the early diagnosis is more important than anything. Diagnosis and treatment for gastric cancer is also following this innovation. Gastric cancer is one of the most common cancer in the world and second most common cause of cancer-related deaths [1$3]$. When gastric cancer is detected in an advanced stage, the 5-year survival rate is low: 10\%-20\% [4]. However, the 5-year survival rates of patients with early gastric cancer, limited to the mucosa or the submucosa, were $99 \%$ and $96 \%$, respectively $[3,5]$. Introducing endoscopic submucosal dissection (ESD) changed the paradigm of gastric cancer treatment. ESD currently has settled as the standard treatment for patients with a lower mortality risk from metastasis as compared to that from surgery [6]. Thus, early diagnosis and early resection have enormous influence on the quality of life and saving stomach, as well as the survival of patients with gastric cancer. Considering recent advancement of therapeutic endoscopic practice, it cannot be more important to emphasize accurate diagnosis of small early lesions.

The advance of optic technology has led the remarkable evolutions in endoscopic images. Currently, the resolution power of white light endoscopy is as high as to detect objects 10-71 microns in diameter, compared with the naked eye which can discriminate objects 125-165 microns in diameter [7]. Adding to this, enhancement technologies including surface enhancement, color enhancement, and edge enhancement were efficiently used to help finding out suspicious mucosal changes more easily [8]. High pixel image and special enhancement technologies are the basis of current endoscopic image system. Magnification endoscopy usually involves the use of a movable lenses that allows the endoscopist to zoom in on a small area of mucosa and magnify it up to 150 times [9]. Narrow Band Image added significant power to magnifying endoscopy for investigating microanatomy of gastrointestinal mucosa. The combination of two technologies produced clinically applicable outcomes for identifying gastric cancer [10-12]. Confocal laser endomicroscopy enables the endoscopist to obtain real-time in vivo histologic images, which is called optical biopsies for gastric cancer [13-15]. However, these advances were only focused on investigating morphologic changes not on biochemical or molecular analysis of target tissues. Currently, positive endoscopic biopsy is the standard criterion for gastric cancer diagnosis, but is invasive and impractical for screening 
high-risk patients who may have multiple suspicious lesions [16]. In addition, endoscopic biopsies are often small (about $3 \mathrm{~mm}$ in diameter) and maybe inconclusive both in making the diagnosis and in determining the type of gastric adenocarcinoma [17]. Additionally, adenomas were frequently revealed to be carcinomas after endoscopic resection [1820]. Furthermore, it is not uncommon that pathologic results are not concordant among pathologists [21]. Considering this circumstance, there is a clear need for an objective imaging diagnostic tool, which relies on biochemical or molecular analysis of target tissues rather than an individual's assessment of cellular appearance.

Raman spectroscopy represents a unique optical vibrational technique based on the fundamental premise of inelastic light scattering for tissue diagnosis and characterization [22-24]. Taking advantage of the Raman spectroscopic ability of harvesting a wealth of fingerprint information from interand/or intracellular components such as proteins, lipids, and DNA in cells and tissue, Raman spectroscopy has shown great promise for histopathologic assessments at the biomolecular level [23-26]. Many investigations have been conducted to reveal the biomolecular fingerprint of gastric cancer compared with normal tissue. In this review, basic principle, biomedical fingerprinting, instrumentation, and clinical implication for gastric cancer of Raman spectroscopy were covered with specific physical explanation.

\section{Principle of Raman Spectroscopy}

Raman spectroscopy is a spectroscopic technique based on inelastic scattering of monochromatic light, usually from a laser source [27]. Inelastic scattering is a phenomenon that the frequency of photons changes upon interaction with a sample. When a molecule is excited by the photons of the laser light and then reemitted photons, frequency of the reemitted photons is shifted up or down compared with original monochromatic frequency. This is called the Raman effect $[28,29]$. This shift of frequency provides unique information of molecules. Monochromatic laser light with frequency $v_{0}$ excites molecules and transforms them into oscillating dipoles. Such oscillating dipoles emit light of three different frequencies when they return to lower energy level [28]. Raman scattering separated into two types: stoke and antistoke. Stoke is a phenomenon that emitted frequency is lower than exciting frequency. A photon with frequency $v_{0}$ excited Raman-active molecule which at the time of interaction is in the basic vibrational state. Part of the photon's energy is transferred to the Raman-active mode with frequency $v_{m}$ and the resulting frequency of scattered light is reduced to $v_{0}-v_{m}$ (Figure 1 ). When an emitted frequency is higher than an excited frequency, this scattering is called as antistoke. A photon with frequency $v_{0}$ excited a Ramanactive molecule, which, at the time of interaction, is already in the excited vibrational state. Excessive energy of excited Raman active mode is released, molecule returns to the basic vibrational state and the resulting frequency of scattered light goes up to $v_{0}+v_{m}$ (Figure 1 ). Only about $0.001 \%$ of the incident photons generates Raman signal with frequencies $v_{0} \pm v_{m}$; approximately $99.999 \%$ of incident light undergo

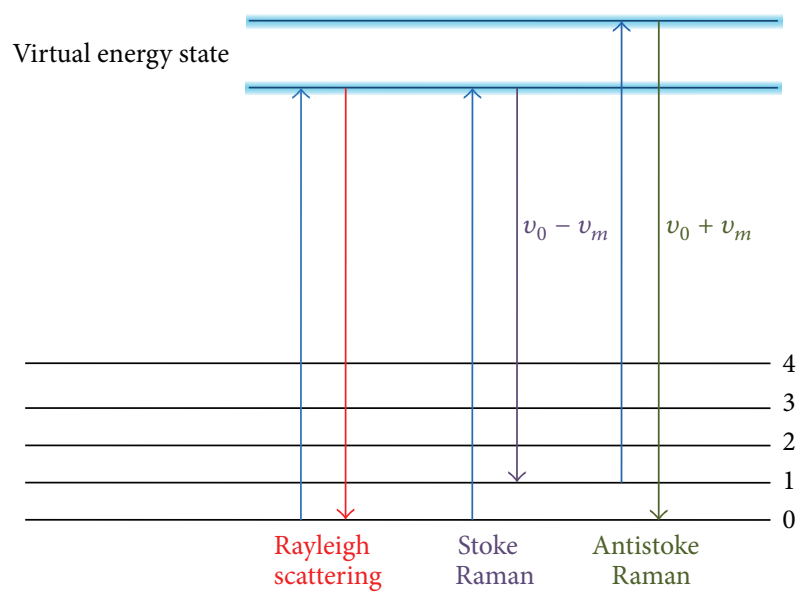

FIGURE 1: Three types of scattering: Rayleigh, Stoke, and Antistoke. Rayleigh scattering is a interaction that the excited molecule returns back to the same basic vibrational state and emits light with the same frequency $v_{0}$ as an excitation source. Raman scattering occurs when a photon with frequency $v_{0}$ is excited by Raman-active molecule. Stoke frequency is generated by the part of the photon energy is transferred with frequency $v_{m}$ and the resulting frequency of scattered light is diminished to $v_{0}-v_{m}$. Antistoke frequency is released when a photon with frequency $v_{0}$ is excited by a Raman active molecule with already excited vibrational state. The energy level goes up to $v_{0}+v_{m}$. Finally released frequency of scattered light from high virtual energy state to the ground state coincides with $v_{0}+v_{m}$.

Rayleigh scattering, but this signal is not useful for molecular characterization; Rayleigh scattering is an elastic interaction between a photon and a molecule; a photon with the frequency $v_{0}$ is absorbed by a molecule with no Raman-active mode. After then, the excited molecule returns back to the same basic vibrational state and emits light with the same frequency $v_{0}$ as an excitation photon.

2.1. Biochemical Fingerprint by Raman Spectroscopy. Raman spectroscopy enables elucidating the biochemical characterization of a tissue through estimating the molecular specific inelastic scattering [27]. Raman spectroscopy needs a monochromatic laser illumination to sample tissue. Subsequently, collection and analysis of the scattered light is required. Photons in the incident laser light undergo inelastic collisions with molecules, causing an exchange of energy and therefore a change in frequency. The frequency change is dependent on the species of molecule. The shift is independent of the wavelength of excitation, which means that the energy shift is constant for each separate molecular species. The intensity of the signal is directly proportional to the concentration of the molecular constituents. Therefore, the Raman spectrum represents molecular composition of the interrogated volume providing a direct molecular fingerprint. Raman spectrum is the series of the scattered light intensity versus its change in frequency. Raman frequency shifts are conventionally measured in wave numbers $\left(\mathrm{cm}^{-1}\right)$. The biological molecule of cholesterol represented by Raman spectroscopy is demonstrated in Figure 2 [27, 31]. Each band of scattered light 


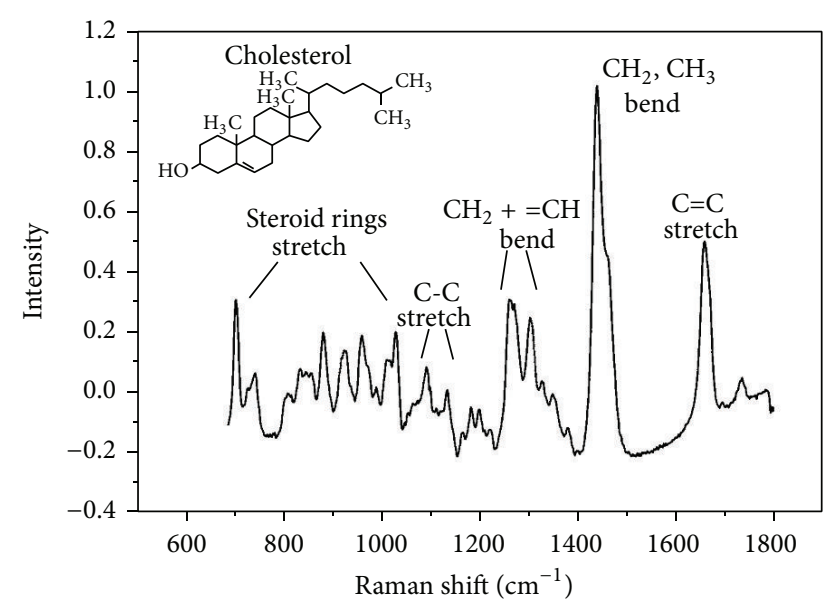

Figure 2: Near-infrared Raman spectrum. Near-infrared Raman spectrum of cholesterol indicating typical vibrational bands. The background has been removed by subtracting a fourth-order polynomial fit to the raw spectrum (adopted from Hanlon et al. Phys Med Biol 2000; 45: R1-59) [27].

of Raman spectrum is the characteristic of specific molecular vibrations, which, taken all together, are unique for cholesterol. For example, the characteristic Raman peak at $1440 \mathrm{~cm}^{-1}$ is caused by the $\mathrm{CH}_{2}$ and $\mathrm{CH}_{3}$ deformation vibrations, and the peak at $1670 \mathrm{~cm}^{-1}$ matches to $\mathrm{C}=\mathrm{C}$ stretching vibrations. If a sample of biological tissue has cholesterol, these peaks will be observed in its Raman spectrum. Thus, the Raman spectrum can provide a "fingerprint" of a substance from which the molecular composition can be determined [27]. Fortunately, the majority of biological molecules are Raman active, each with their own fingerprint [32]. As a result, Raman spectroscopy is a very sensitive tool to subtle biochemical and molecular changes, which is crucial for differentiating tissues (Figure 3). Additionally, water, the predominant component of living tissue, gives a negligible Raman signal due to the limited change in polarisability of the $-\mathrm{OH}$ bond. This, thus, enables analysis of fresh, unprepared tissue, both in vitro and in vivo [33]. These properties make Raman spectroscopy potentially a very powerful diagnostic tool.

\subsection{Basic Instrumentation. Spontaneous Raman scattering is} very weak and special measures should be taken to distinguish it from the predominant Rayleigh scattering [27]. A Raman spectroscopy system typically consists of four major components: illumination source and system (laser), light collection optics, wavelength selector (filter or spectrophotometer), detector (photodiode array, charge coupled device or photomultiplier tube) (Figure 4). A lens collects the light, and a filter separates the Raman scatterings from the incident light. These shifted wavelengths then traverse the monochromator and detection system which measures their frequency. The specific frequencies of the shifted scattered light reveal the molecular structure of the sample material. Aside from Rayleigh scattering, fluorescence phenomenon is another hurdle to overcome for obtaining precise Raman scattering signal because the intensity of fluorescence is very strong and

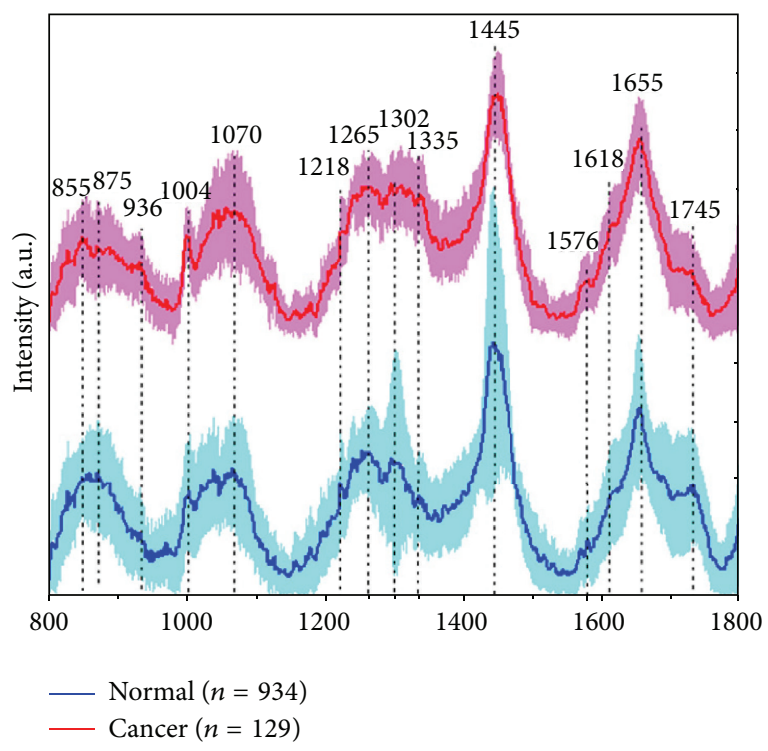

FIGURE 3: In vivo mean Raman spectra \pm 1 standard deviations of normal ( $n=934)$, and cancer $(n=129)$ gastric tissue (adopted from Huang et al. Biosens Bioelectron 2010; 26: 383-389) [30].

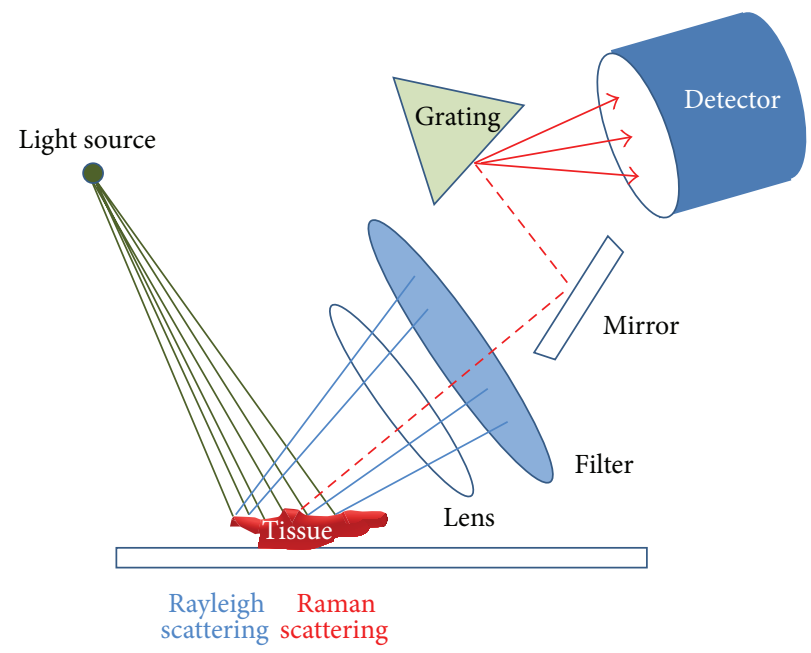

FIgURE 4: Schematic presentation of Raman spectroscopy instrument. When excitation light irradiates (green line), Rayleigh scattering (blue line) and Raman scattering (red dotted line) are released. Rayleigh scattering is filtered, and pure Raman scattering is detected.

the signal spectrums overlap with Raman scattering. Using near infrared excitation is an excellent solution because it minimizes spectral disruption from tissue fluorescence [33]. Furthermore, near infrared light has less mutagenic effect and deeper penetration capability than other light sources. Ultraviolet-resonance Raman spectroscopy can be another option because fluorescence spectrum is separated from Raman spectrum when ultraviolet ray (wavelength $<270 \mathrm{~nm}$ ) was illuminated $[34,35]$. However, this method can cause mutagenesis and depth of tissue penetration is seriously limited. The combination of Raman spectroscopy and wide 


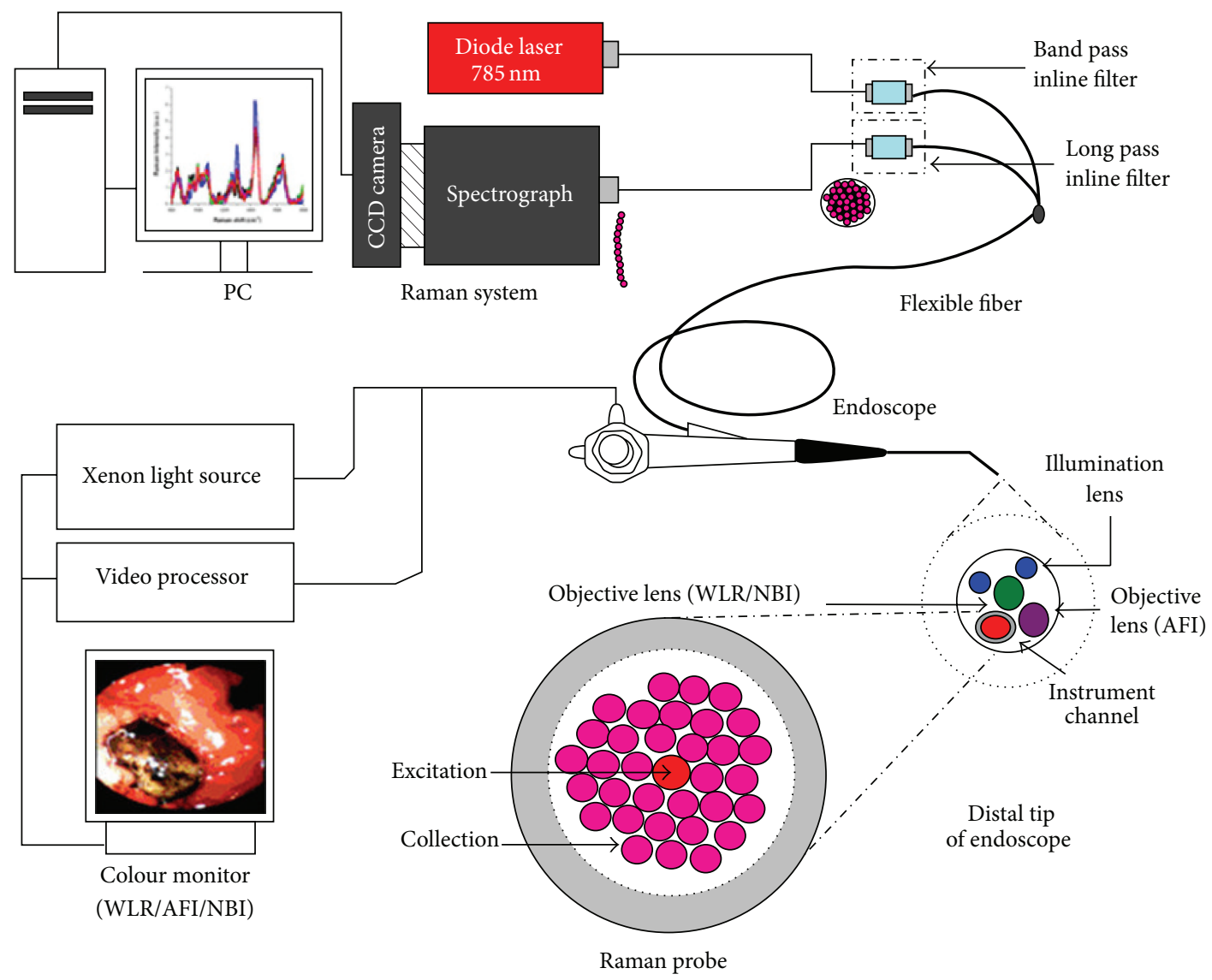

FIGURE 5: Schematic of the image-guided Raman endoscopy system for in vivo tissue Raman measurements during clinical gastroscopy. WLR, white light reflectance; AFI, autofluorescence imaging; NBI, narrow-band imaging (adopted from Huang et al. Biosens Bioelectron 2010; 26: 383-389) [30].

field endoscopic system used by Huang et al. was depicted in Figure 5.

\section{Clinical Implementation}

Huang et al. conducted many investigations to reveal the fingerprint of gastric cancer and dysplasia developing applicable algorithms $[24,30,36-43]$. This system consists of a 785-nm diode laser, a transmissive spectrograph with a Kaiser holographic grating, an near infrared-optimized backilluminated, deep-depletion charge-coupled device detector (Princeton Instruments, Trenton, NJ, USA), and an in-house developed fiber-optic Raman probe that can effectively eliminate interference from fiber-optic fluorescence and silica Raman signals [44]. They fabricated a beveled fiber-optic confocal Raman probe coupled with a ball lens for enhancing in vivo epithelial tissue Raman measurements at endoscopy [25]. The confocal Raman probe design can be optimized for maximizing shallower tissue Raman measurements in epithelial tissue; in addition, the ratio of epithelium to stromal Raman photons collected using an optimized confocal Raman probe is approximately 19 -fold higher than that using a volume-type Raman probe. The system acquired Raman spectra over the wave number range of 800 to $1800 \mathrm{~cm}^{-1}$, and each spectrum was acquired within $5 \mathrm{~s}$ with light irradiance of $1.56 \mathrm{Wcm}^{-2}$. The spectral resolution of the system is $4 \mathrm{~cm}^{-1}$. This group developed fully automated on-line Raman spectral diagnostics framework integrated with a multimodal image-guided Raman technique for real-time in vivo cancer detection at endoscopy [45]. Prior to developing on-line system, data-analysis has mostly been limited to post-processing and off-line algorithm development.

3.1. Ex Vivo Investigation. Teh et al. analyzed 76 gastric samples, 55 normal and 21 dysplasia, from 44 patients who underwent gastrectomy or endoscopic biopsies with clinically suspicious lesions. There are specific differences in Raman spectra between normal and dysplasia tissue, particularly in the spectral ranges of $1200-1500 \mathrm{~cm}^{-1}$ and $1600-1800 \mathrm{~cm}^{-1}$, which contained signals related to amide III and amide I of proteins, $\mathrm{CH}_{3} \mathrm{CH}_{2}$ twisting of proteins/nucleic acids, and the $\mathrm{C}=\mathrm{C}$ stretching mode of phospholipids, respectively. The normal tissue showed Raman peak intensity at $875 \mathrm{~cm}^{-1}$ whereas the dysplasia demonstrated peak intensity at $1450 \mathrm{~cm}^{-1}$. Diagnostic algorithm based on principal components analysis (PCA) and linear discriminant analysis (LDA) yielded the diagnostic sensitivity of $95.2 \%$ and specificity $90.9 \%$ for separating dysplasia from normal gastric tissue [37]. Regarding 


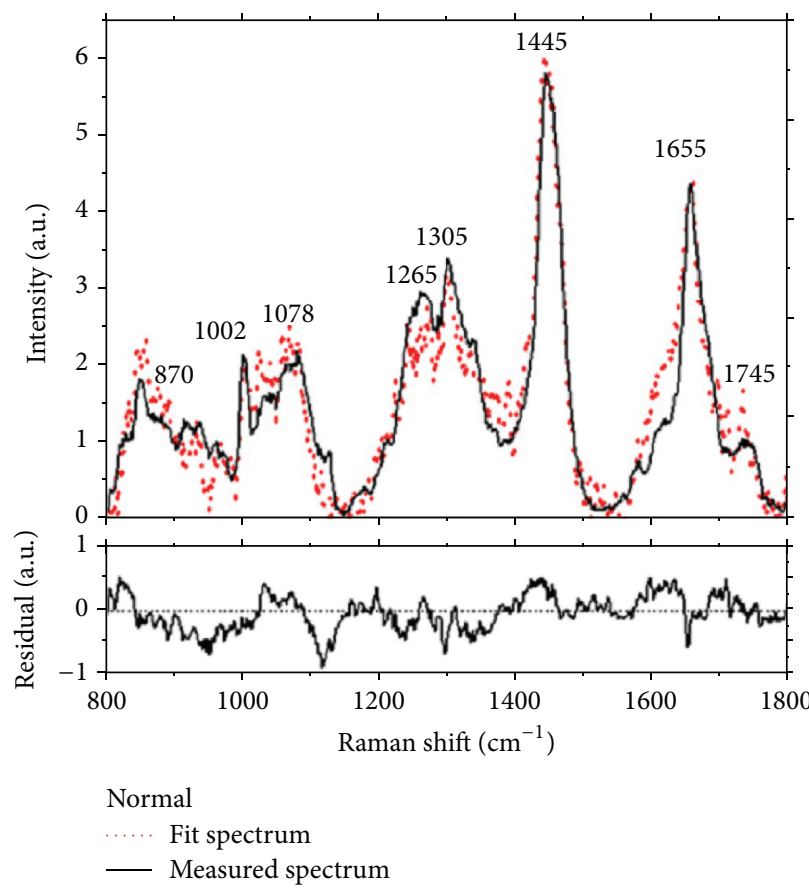

(a)

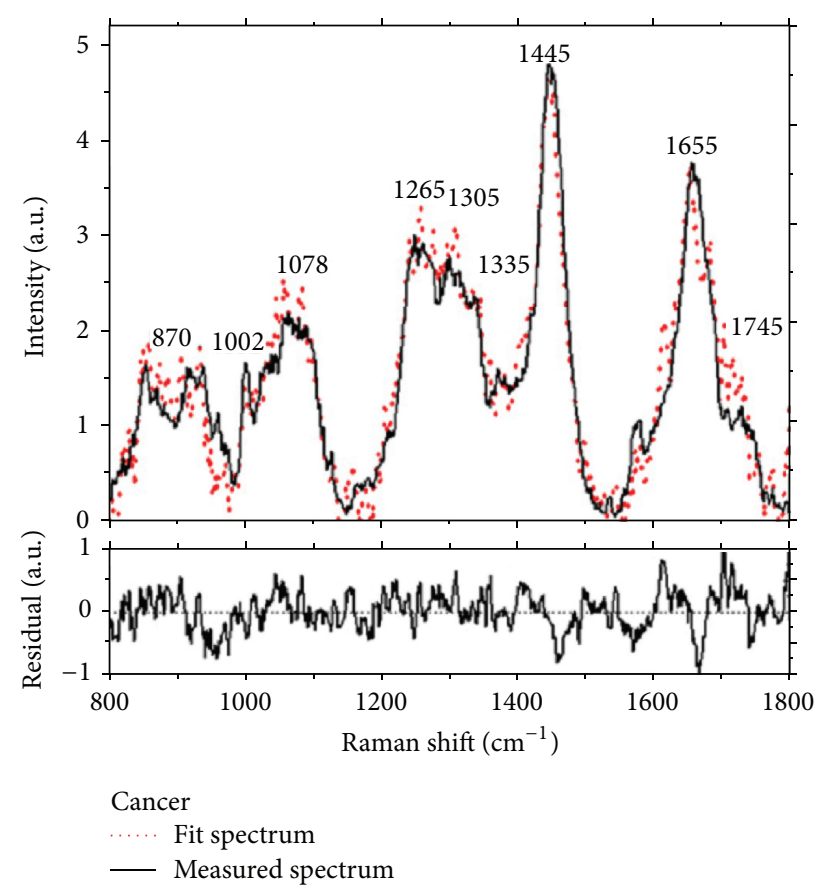

(b)

Figure 6: Comparison of in vivo gastric Raman spectra measured with the reconstructed tissue Raman spectra through the employment of the eight basis reference Raman spectra: (a) normal, and (b) cancerous gastric tissue. Residual of the fits are also shown on the same scale in each plot (adopted from Huang et al. Biosens Bioelectron 2010; 26: 383-389) [30].

differentiating gastric cancer from normal tissue, classification and regression tree, based on the recursive partitioning for classification of different subgroups in complex datasets, was introduced [36]. Raman peaks at $875 \mathrm{~cm}^{-1}$ and $1450 \mathrm{~cm}^{-1}$ were demonstrated in normal and dysplastic tissue, respectively, and the diagnostic sensitivity and specificity of learning dataset were $90.2 \%$ and $95.7 \%$; the predictive sensitivity and specificity of the independent validation dataset were $88.9 \%$ and $92.9 \%$ (55 normal and 18 cancer samples) [36]. In 2013, Luo et al. demonstrated substantial difference among normal tissue, adenoma, and adenocarcinoma. They employed diverse statistical methods to develop effective diagnostic algorithms for classifying the Raman spectra of different types of ex vivo gastric tissues, including PCA, LDA, and naive Bayesian classifier (NBC) techniques. Compared with PCA-LDA algorithms, PCA and NBC techniques together with leave-one-out, cross-validation method provide better discriminative results of normal, adenoma, and adenocarcinoma gastric tissues, resulting in sensitivities of $96.3 \%$, $96.9 \%$, and $96.9 \%$, and specificities of $93 \%, 100 \%$, and $95.2 \%$, respectively.

3.2. In Vivo Precancerous Lesion and Adenocarcinoma Study. The in vivo Raman spectroscopy study of gastric dysplasia and normal tissue was reported by Huang et al. combined with wide field endoscopy system, narrow band image, in 2010 [41]. There were significant differences between normal $(n=$ $54)$ and dysplastic $(n=18)$ gastric tissue from 30 patients, particularly in the spectral ranges of 825 to 950,1000 to 1100 ,
1250 to 1500 , and 1600 to $1800 \mathrm{~cm}^{-1}$. Multivariate analysis analysis, based on PCA and LDA, together with the leaveone tissue site-out, cross validation on in vivo gastric Raman spectra yielded a sensitivity of $94.4 \%$ (17/18) and specificity of $96.3 \%$ (52/54) for distinction of gastric dysplastic tissue [41].

The first in vivo study of gastric cancer was firstly presented in 2010 also. [30]. They revealed that the fit coefficients from albumin, nucleic acid, phospholipids and histones were found to be the most substantial features for construction of the diagnostic model, giving rise to an overall accuracy of $93.7 \%$, sensitivity of $94.0 \%$ (110/117), and specificity of $93.4 \%$ (113/121) for in vivo discrimination of gastric cancer from normal gastric tissue: result from 1063 in vivo Raman spectra from 238 tissue sites of 67 patients, 121 normal tissue and 117 gastric cancer tissue (Figure 6) [30]. Subsequent study, 238 gastric tissues from 67 patients, using ant colony optimization (ACO) integrated with LDA algorithm identified seven diagnostically important Raman bands in the regions of 850 to 875,1090 to 1110,1120 to 1130,1170 to 1190,1320 to 1340,1655 to 1665 and 1730 to $1745 \mathrm{~cm}^{-1}$ provided a diagnostic sensitivity of $94.6 \%$ and specificity of $94.6 \%$ for distinction of gastric cancer [26].

The carcinogenesis of gastric cancer includes several premalignant cascade processes from chronic atrophic gastritis to intestinal metaplasia and dysplasia [46]. Bergholt et al. assessed the capability of Raman spectroscopy for multiclass elucidation of intestinal-type gastric carcinogenesis sequence in vivo. Raman spectroscopy integrated with semi-quantitative spectral modeling (e.g., DNA, lipids, glycoprotein, 
proteins and blood) reveals the progressive changes of biochemical constituents in gastric tissue associated with preneoplastic and neoplastic transformation [26]. Multiclass probabilistic partial least square-discriminant analysis (PLSDA) diagnostic algorithms based on in vivo Raman spectra are able to identify normal mucosa with sensitivity of $75.88 \%$ and specificity of $87.21 \%$; intestinal metaplasia with sensitivity of $46.67 \%$ and specificity of $87.55 \%$; dysplasia with sensitivity of $83.33 \%$; specificity of $96.80 \%$, and adenocarcinoma with sensitivity of $84.91 \%$ and specificity of $95.57 \%$. This study probed that Raman spectroscopy is a sensitive biomolecular probe for monitoring intestinal-type gastric carcinogenesis to realize early diagnosis and detection of precancerous lesions as well as gastric cancer. In vivo studies demonstrated that image-guided Raman endoscopy technique has promising potential for the noninvasive, in vivo diagnosis and detection of gastric cancer and dysplasia at the molecular level.

In clinical field, differentiating malignant ulcers from benign ulcers is quite important. However, this is not an easy task using conventional technique. Bergholt et al. demonstrated Raman spectroscopy can help clinicians in this point [39]. A total of 1102 Raman spectra were acquired from 71 patients with gastric ulcers (111 Raman spectra from benign ulcers, 67 Raman spectra from malignant ulcers, 924 Raman spectra from normal tissue). Distinctive spectral differences were observed in Raman spectra among three different tissues; particular spectral ranges were 800 to 900,1000 to 1100,1245 to 1335,1440 to 1450 and 1500 to $1800 \mathrm{~cm}^{-1}$. Raman signal of a malignant ulcer is mainly associated with abnormal nuclear activity and decrease in lipids as compared to a benign ulcer. The partial least squares-discriminant algorithm together with leave on tissue site-out, cross validation technique yielded diagnostic sensitivities of $90.8 \%, 84.7 \%$, and $82.1 \%$, and specificities of $93.8 \%, 94.5 \%$, and $95.3 \%$, respectively, for classification of normal mucosa, benign ulcers, and malignant ulcerous lesions in the stomach [39].

The distinction between intestinal and diffuse types of gastric adenocarcinoma is clinically relevant and may influence treatment strategy [2]. The clinical potential of near infrared Raman spectroscopy for identifying different subtypes of gastric adenocarcinoma was reported by Teh et al. in 2010 [42]. There were significant differences in Raman spectra between normal stomach and the two gastric adenocarcinoma subtypes, particularly in the spectral ranges 850$1150,1200-1500$, and $1600-1750 \mathrm{~cm}^{-1}$, which contain signals related to proteins, nucleic acids and lipids (Figure 7). The predictive accuracies were of $88 \%$ with $92 \%$. $94 \%$ for normal stomach, intestinal type adenocarcinoma, diffuse type adenocarcinoma, respectively. This result was reproduced by Kawabata et al's investigation [47].

3.3. Real Time on Line System. As above investigations presented, Raman spectroscopy is a notable method for understanding precancerous condition and adenocarcinoma in the stomach. However, data-analysis has been mostly performed after obtaining Raman spectrum in off line algorithm establishment before a fully automated on-line Raman spectral diagnostic system was developed for real-time in vivo cancer

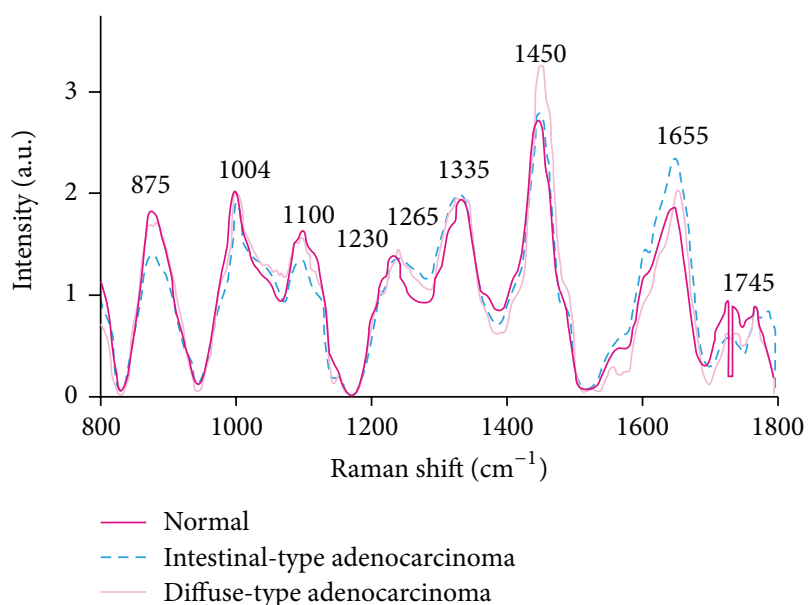

(a)

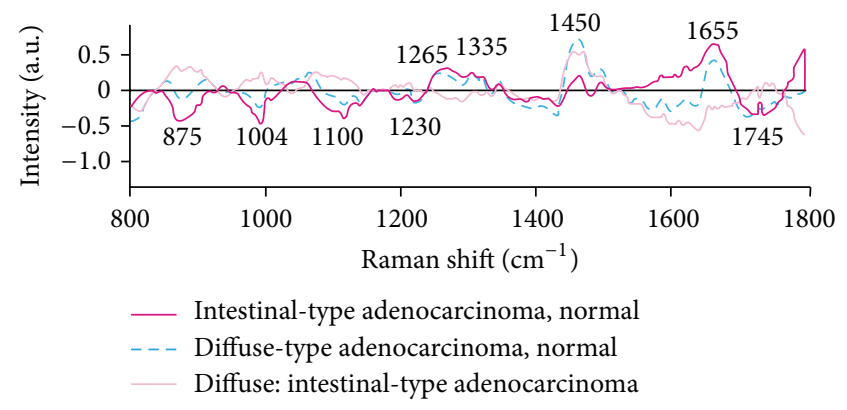

(b)

FIgURE 7: Comparison of mean near-infrared Raman spectra of 70 normal stomach samples, (a) 18 intestinal-type adenocarcinomas and 12 diffuse-type adenocarcinomas. (b) Difference spectra were calculated from the mean Raman spectra among the three gastric tissue types (adopted from Teh et al. Br J Surg 2010; 97: 550-557) [42].

detection at endoscopy [45]. They tested this system with a total of 2748 in vivo gastric tissue spectra (2465 normal and 283 cancer) from 305 patients [45]. Free-running optical diagnosis and processing time of no more than $0.5 \mathrm{~s}$ can be achieved, which is critical to realizing real-time in vivo tissue diagnostics during clinical endoscopic examination [45]. The optimized partial least squares-discriminant analysis (PLS-DA) models based on the randomly resampled training database provide the diagnostic accuracy of $85.6 \%$, sensitivity of $80.5 \%$, and specificity of $86.2 \%$ for the detection of gastric cancer. This on-line real time endoscopic Raman spectroscopy definitely opened a door for clinical application.

\section{Conclusion}

The goal of endoscopic Raman spectroscopy in the stomach is to accurate early diagnosis of gastric cancer which can be under or over diagnosed by conventional methods. Early accurate diagnosis provides a minimally invasive and minimally destructive treatment such as ESD, so the quality of patient is dramatically improved compared with surgical treatment. However, the accurate diagnosis of small obscure 
lesion is not easy in conventional method including forcepsbiopsy. Several investigations demonstrated that adenoma was frequently revealed to be carcinoma after endoscopic resection within the range of $6 \%$ to $47 \%$ [18-20]. This discrepancy between forceps-biopsied specimen and postresection specimen is inevitable limitation of forceps biopsy due to the lack of specimens [48-51]; especially, in the case of ESD, forceps-biopsy number is often significantly limited in order to prevent possible fibrosis that multiple forcepsbiopsies can accompany. In this perspective, endoscopic Raman spectroscopy has a substantial potential in several points combined with wide filed technologies.

The most prominent feature of Raman spectroscopy is objective, reliable, and reproducible histologic estimation by molecular analysis making Raman spectroscopy different from other optical biopsy technologies such as confocal endomicroscopy. Confocal endomicroscopy is currently applied in usual clinical practice and produced several results regarding gastric cancer verification [52-54]. However, confocal endomicroscopy clearly needs long learning curve for maneuvering an endoscopy and probe to acquire high quality images although training for reading acquired picture may not be difficult $[55,56]$. The presence of moving artifacts caused by breathing and heart beat disturb obtaining high quality images. Furthermore, there are similar problems that lurk in pathologic evaluation; interobserver discrepancy. Contrasting to other optical biopsy technologies, Raman spectroscopy automatically analyze the summation of specific molecular components in a target tissue and provide objective and reproducible diagnostic value through complicated algorithms [26, 30, 39, 41, 44, 45, 47]. This is a significant edge of Raman spectroscopy, which enables us to evade interand intra-observer discrepancies and to make consistent therapeutic plans for patients with same disease.

There are also limitations in Raman spectroscopy. First, Raman spectroscopy only identifies molecular features. Thus if two molecules have the same molecular features, they may be indistinguishable from each other, for instance, arachidonic acid and eicosapentaenoic acid. Second, fluorescence of impurities or of the sample itself can hide the Raman spectrum. Third, sample heating through the intense laser radiation can destroy the sample or cover the Raman spectrum.

Adding to diagnosing disease, Raman spectroscopy can be applied for understanding the margin of the cancer for obtaining the ideal resection area also. Following ESD, Raman spectroscopy can scan healed ulcer to confirm no cancer recurrence, especially when hypertrophic scar is noticed. This technology can guide accurate target biopsy instead of multiple forceps-biopsies. Furthermore, small erosions, frequently observed in elderly patients with anticoagulation therapy, might be easily elucidated with Raman spectroscopy without forceps-biopsy. For these applications, many prospective investigations would be necessary to verify its capability. Increased accuracy with developing updated algorithms and developing on-line real time automatic analysis system clearly opened a way to apply Raman spectroscopy for clinicians. Raman spectroscopy is expected to provide us with accurate, objective, and reliable diagnosis and may have potential to replace multiple forceps biopsies.

\section{Conflict of Interests}

The author declares that he has no conflict of interests.

\section{Acknowledgment}

This research was supported by Basic Science Research Program through the National Research Foundation of Korea (NRF) funded by the Ministry of Science, ICT and Future Planning (NRF-2013R1A1A1009682). The author appreciates Professor Kenshi Yao and Dr. Noriya Uedo for guidance to the world of image anaylsis.

\section{References}

[1] L. Yang, "Incidence and mortality of gastric cancer in China," World Journal of Gastroenterology, vol. 12, no. 1, pp. 17-20, 2006.

[2] B. J. Dicken, D. L. Bigam, C. Cass, J. R. Mackey, A. A. Joy, and S. M. Hamilton, "Gastric adenocarcinoma: review and considerations for future directions," Annals of Surgery, vol. 241, no. 1, pp. 27-39, 2005.

[3] K. D. Crew and A. I. Neugut, "Epidemiology of gastric cancer," World Journal of Gastroenterology, vol. 12, no. 3, pp. 354-362, 2006.

[4] X.-M. Zou, Y.-L. Li, H. Wang et al., "Gastric cancer cell lines induced by trichostatin A," World Journal of Gastroenterology, vol. 14, no. 30, pp. 4810-4815, 2008.

[5] T. Sano, M. Sasako, T. Kinoshita, and K. Maruyama, "Recurrence of early gastric cancer: follow-up of 1475 patients and review of the Japanese literature," Cancer, vol. 72, no. 11, pp. 3174-3178, 1993.

[6] K. Ludwig, G. Klautke, J. Bernhard, and R. Weiner, "Minimally invasive and local treatment for mucosal early gastric cancer," Surgical Endoscopy and Other Interventional Techniques, vol. 19, no. 10, pp. 1362-1366, 2005.

[7] S. C. Reddymasu and P. Sharma, "Advances in endoscopic imaging of the esophagus," Gastroenterology Clinics of North America, vol. 37, no. 4, pp. 763-774, 2008.

[8] S. Kodashima and M. Fujishiro, "Novel image-enhanced endoscopy with i-scan technology," World Journal of Gastroenterology, vol. 16, no. 9, pp. 1043-1049, 2010.

[9] I. Waxman and V. J. Konda, "Endoscopic techniques for recognizing neoplasia in Barrett's esophagus: which should the clinician use?" Current Opinion in Gastroenterology, vol. 26, no. 4, pp. 352-360, 2010.

[10] M. Kaise, M. Kato, M. Urashima et al., "Magnifying endoscopy combined with narrow-band imaging for differential diagnosis of superficialdepressed gastric lesions," Endoscopy, vol. 41, no. 4, pp. 310-315, 2009.

[11] Y. Ezoe, M. Muto, T. Horimatsu et al., "Magnifying narrowband imaging versus magnifying white-light imaging for the differential diagnosis of gastric small depressive lesions: a prospective study," Gastrointestinal Endoscopy, vol. 71, no. 3, pp. 477-484, 2010.

[12] M. Kato, M. Kaise, J. Yonezawa et al., "Magnifying endoscopy with narrow-band imaging achieves superior accuracy in the differential diagnosis of superficial gastric lesions identified with white-light endoscopy: a prospective study," Gastrointestinal Endoscopy, vol. 72, no. 3, pp. 523-529, 2010.

[13] K. Banno, Y. Niwa, R. Miyahara et al., "Confocal endomicroscopy for phenotypic diagnosis of gastric cancer," Journal 
of Gastroenterology and Hepatology, vol. 25, no. 4, pp. 712-718, 2010.

[14] S. Kitabatake, Y. Niwa, R. Miyahara et al., "Confocal endomicroscopy for the diagnosis of gastric cancer in vivo," Endoscopy, vol. 38, no. 11, pp. 1110-1114, 2006.

[15] M. J. Antunes, "Requiem for a good mechanical heart valve: farewell to the Medtronic Hall valve," The Journal of Thoracic and Cardiovascular Surgery, 2015.

[16] R. Jorde, H. Ostensen, L. H. Bostad, P. G. Burhol, and F. T. Langmark, "Cancer detection in biopsy specimens taken from different types of gastric lesions," Cancer, vol. 58, no. 2, pp. 376382, 1986.

[17] Y. Matsubara, H. Yanai, K. Ishiguro et al., "Clinical interpretation of the histological typing of gastric cancer using endoscopic forceps biopsy," Hepatogastroenterology, vol. 51, no. 55, pp. 285288, 2004.

[18] K. Elster, "Histologic classification of gastric polyps," Current Topics in Pathology, vol. 63, pp. 77-93, 1976.

[19] T. Nakamura and G. I. Nakano, "Histopathological classification and malignant change in gastric polyps," Journal of Clinical Pathology, vol. 38, no. 7, pp. 754-764, 1985.

[20] T. Kamiya, T. Morishita, H. Asakura, S. Miura, Y. Munakata, and M. Tsuchiya, "Long-term follow-up study on gastric adenoma and its relation to gastric protruded carcinoma," Cancer, vol. 50, no. 11, pp. 2496-2503, 1982.

[21] R. J. Schlemper, R. H. Riddell, Y. Kato et al., "The vienna classification of gastrointestinal epithelial neoplasia," Gut, vol. 47, no. 2, pp. 251-255, 2000.

[22] C. V. Raman and K. S. Krishnan, "A new type of secondary radiation," Nature, vol. 121, no. 3048, pp. 501-502, 1928.

[23] M. G. Shim, L.-M. Wong Kee Song, N. E. Marcon, and B. C. Wilson, "In vivo near-infrared raman spectroscopy: demonstration of feasibility during clinical gastrointestinal endoscopy," Photochemistry and Photobiology, vol. 72, no. 1, pp. 146-150, 2000.

[24] Z. Huang, S. K. Teh, W. Zheng et al., "Integrated Raman spectroscopy and trimodal wide-field imaging techniques for real-time in vivo tissue Raman measurements at endoscopy," Optics Letters, vol. 34, no. 6, pp. 758-760, 2009.

[25] J. Wang, M. S. Bergholt, W. Zheng, and Z. Huang, "Development of a beveled fiber-optic confocal Raman probe for enhancing in vivo epithelial tissue Raman measurements at endoscopy," Optics Letters, vol. 38, no. 13, pp. 2321-2323, 2013.

[26] M. S. Bergholt, W. Zheng, K. Y. Ho et al., "Fiber-optic Raman spectroscopy probes gastric carcinogenesis in vivo at endoscopy," Journal of Biophotonics, vol. 6, no. 1, pp. 49-59, 2013.

[27] E. B. Hanlon, R. Manoharan, T.-W. Koo et al., "Prospects for in vivo Raman spectroscopy," Physics in Medicine and Biology, vol. 45, no. 2, pp. R1-R59, 2000.

[28] H. Tsuda and J. Arends, "Raman spectroscopy in dental research: a short review of recent studies," Advances in Dental Research, vol. 11, no. 4, pp. 539-547, 1997.

[29] R. S. Dacosta, B. C. Wilson, and N. E. Marcon, "New optical technologies for earlier endoscopic diagnosis of premalignant gastrointestinal lesions," Journal of Gastroenterology and Hepatology, vol. 17, no. 1, pp. S85-S104, 2002.

[30] Z. Huang, S. K. Teh, W. Zheng et al., "In vivo detection of epithelial neoplasia in the stomach using image-guided Raman endoscopy," Biosensors and Bioelectronics, vol. 26, no. 2, pp. 383389, 2010.
[31] M. Diem, A. Mazur, K. Lenau et al., "Molecular pathology via IR and Raman spectral imaging," Journal of Biophotonics, vol. 6 , no. 11-12, pp. 855-886, 2013.

[32] C. Kallaway, L. M. Almond, H. Barr et al., "Advances in the clinical application of Raman spectroscopy for cancer diagnostics," Photodiagnosis and Photodynamic Therapy, vol. 10, no. 3, pp. 207-219, 2013.

[33] L. M. Almond, J. Hutchings, N. Shepherd, H. Barr, N. Stone, and C. Kendall, "Raman spectroscopy: a potential tool for early objective diagnosis of neoplasia in the oesophagus," Journal of Biophotonics, vol. 4, no. 10, pp. 685-695, 2011.

[34] Q. Wu, T. Hamilton, W. H. Nelson, S. Elliott, J. F. Sperry, and M. $\mathrm{Wu}$, "UV Raman spectral intensities of E. coli and other bacteria excited at 228.9, 244.0, and $248.2 \mathrm{~nm}$," Analytical Chemistry, vol. 73, no. 14, pp. 3432-3440, 2001.

[35] S. A. Asher, "UV resonance Raman spectroscopy for analytical, physical, and biophysical chemistry. Part 2," Analytical Chemistry, vol. 65, no. 4, pp. 201A-210A, 1993.

[36] S. K. Teh, W. Zheng, K. Y. Ho, M. Teh, K. G. Yeoh, and Z. Huang, "Diagnosis of gastric cancer using near-infrared Raman spectroscopy and classification and regression tree techniques," Journal of Biomedical Optics, vol. 13, no. 3, Article ID 034013, 2008.

[37] S. K. Teh, W. Zheng, K. Y. Ho, M. Teh, K. G. Yeoh, and Z. Huang, "Diagnostic potential of near-infrared Raman spectroscopy in the stomach: differentiating dysplasia from normal tissue," British Journal of Cancer, vol. 98, no. 2, pp. 457-465, 2008.

[38] M. S. Bergholt, W. Zheng, K. Lin et al., "Characterizing variability in in vivo Raman spectra of different anatomical locations in the upper gastrointestinal tract toward cancer detection," Journal of Biomedical Optics, vol. 16, no. 3, Article ID 037003, 2011.

[39] M. S. Bergholt, W. Zheng, K. Lin et al., "Raman endoscopy for in vivo differentiation between benign and malignant ulcers in the stomach," Analyst, vol. 135, no. 12, pp. 3162-3168, 2010.

[40] M. S. Bergholt, W. Zheng, K. Lin et al., "In vivo diagnosis of gastric cancer using Raman endoscopy and ant colony optimization techniques," International Journal of Cancer, vol. 128, no. 11, pp. 2673-2680, 2011.

[41] Z. Huang, M. S. Bergholt, W. Zheng et al., "In vivo early diagnosis of gastric dysplasia using narrow-band image-guided Raman endoscopy," Journal of Biomedical Optics, vol. 15, no. 3, Article ID 037017, 2010.

[42] S. K. Teh, W. Zheng, K. Y. Ho, M. Teh, K. G. Yeoh, and Z. Huang, "Near-infrared Raman spectroscopy for early diagnosis and typing of adenocarcinoma in the stomach," British Journal of Surgery, vol. 97, no. 4, pp. 550-557, 2010.

[43] S. K. Teh, W. Zheng, K. Y. Ho, M. Teh, K. G. Yeoh, and Z. Huang, "Near-infrared Raman spectroscopy for optical diagnosis in the stomach: identification of Helicobacter-pylori infection and intestinal metaplasia," International Journal of Cancer, vol. 126, no. 8, pp. 1920-1927, 2010.

[44] Z. Huang, H. Lui, X. K. Chen, A. Alajlan, D. I. McLean, and H. Zeng, "Raman spectroscopy of in vivo cutaneous melanin," Journal of Biomedical Optics, vol. 9, no. 6, pp. 1198-1205, 2004.

[45] S. Duraipandian, M. S. Bergholt, W. Zheng et al., "Real-time Raman spectroscopy for in vivo, online gastric cancer diagnosis during clinical endoscopic examination," Journal of Biomedical Optics, vol. 17, no. 8, Article ID 081418, 2012.

[46] P. Correa, "A human model of gastric carcinogenesis," Cancer Research, vol. 48, no. 13, pp. 3554-3560, 1988. 
[47] T. Kawabata, H. Kikuchi, S. Okazaki et al., "Near-infrared multichannel Raman spectroscopy with a $1064 \mathrm{~nm}$ excitation wavelength for ex vivo diagnosis of gastric cancer," Journal of Surgical Research, vol. 169, no. 2, pp. el37-e143, 2011.

[48] W. Remmele and E. F. Kolb, "Malignant transformation of hyperplasiogenic polyps of the stomach. Case report," Endoscopy, vol. 10, no. 1, pp. 63-65, 1978.

[49] G. G. Ginsberg, F. H. Al-Kawas, D. E. Fleischer, H. F. Reilly, and S. B. Benjamin, "Gastric polyps: relationship of size and histology to cancer risk," American Journal of Gastroenterology, vol. 91, no. 4, pp. 714-717, 1996.

[50] S. M. Muehldorfer, M. Stolte, P. Martus, E. G. Hahn, and C. Ell, "Diagnostic accuracy of forceps biopsy versus polypectomy for gastric polyps: a prospective multicentre study," Gut, vol. 50, no. 4, pp. 465-470, 2002.

[51] W. J. Yoon, D. H. Lee, Y. J. Jung et al., "Histologic characteristics of gastric polyps in Korea: emphasis on discrepancy between endoscopic forceps biopsy and endoscopic mucosal resection specimen," World Journal of Gastroenterology, vol. 12, no. 25, pp. 4029-4032, 2006.

[52] J.-N. Zhang, Y.-Q. Li, Y.-A. Zhao et al., "Classification of gastric pit patterns by confocal endomicroscopy," Gastrointestinal Endoscopy, vol. 67, no. 6, pp. 843-853, 2008.

[53] Y.-T. Guo, Y.-Q. Li, T. Yu et al., "Diagnosis of gastric intestinal metaplasia with confocal laser endomicroscopy in vivo: a prospective study," Endoscopy, vol. 40, no. 7, pp. 547-553, 2008.

[54] L. G. Lim, K. G. Yeoh, M. Salto-Tellez et al., "Experienced versus inexperienced confocal endoscopists in the diagnosis of gastric adenocarcinoma and intestinal metaplasia on confocal images," Gastrointestinal Endoscopy, vol. 73, no. 6, pp. 1141-1147, 2011.

[55] T. Kuiper, R. Kiesslich, C. Ponsioen, P. Fockens, and E. Dekker, "The learning curve, accuracy, and interobserver agreement of endoscope-based confocal laser endomicroscopy for the differentiation of colorectal lesions," Gastrointestinal Endoscopy, vol. 75, no. 6, pp. 1211-1217, 2012.

[56] R. Pittayanon, R. Rerknimitr, N. Wisedopas et al., “The learning curve of gastric intestinal metaplasia interpretation on the images obtained by probe-based confocal laser endomicroscopy," Diagnostic and Therapeutic Endoscopy, vol. 2012, Article ID 278045, 6 pages, 2012. 


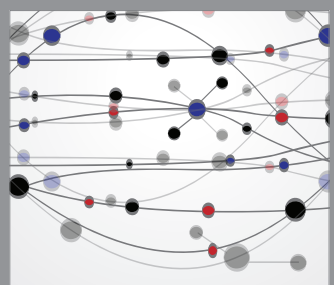

The Scientific World Journal
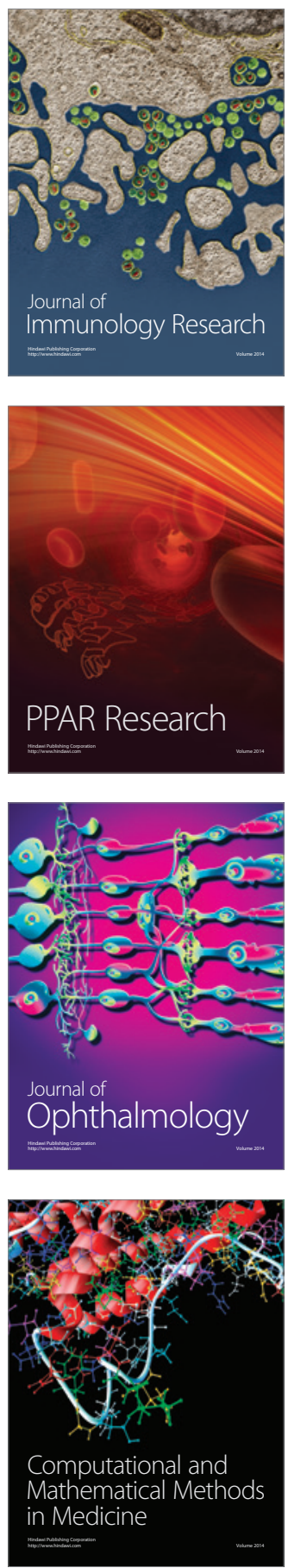

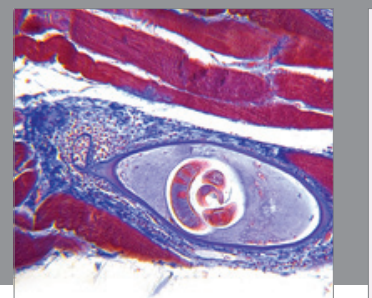

Gastroenterology

Research and Practice
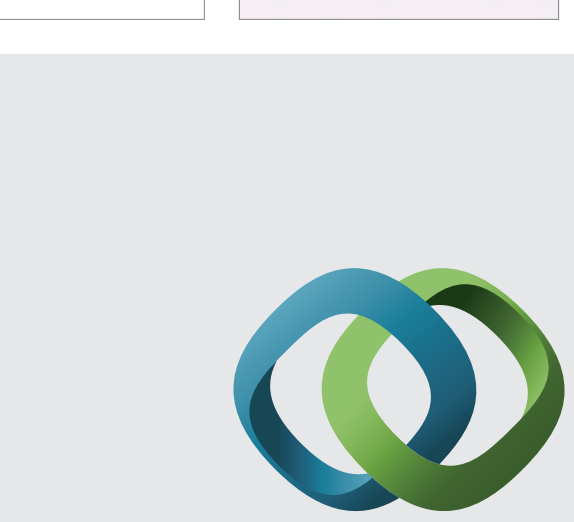

\section{Hindawi}

Submit your manuscripts at

http://www.hindawi.com
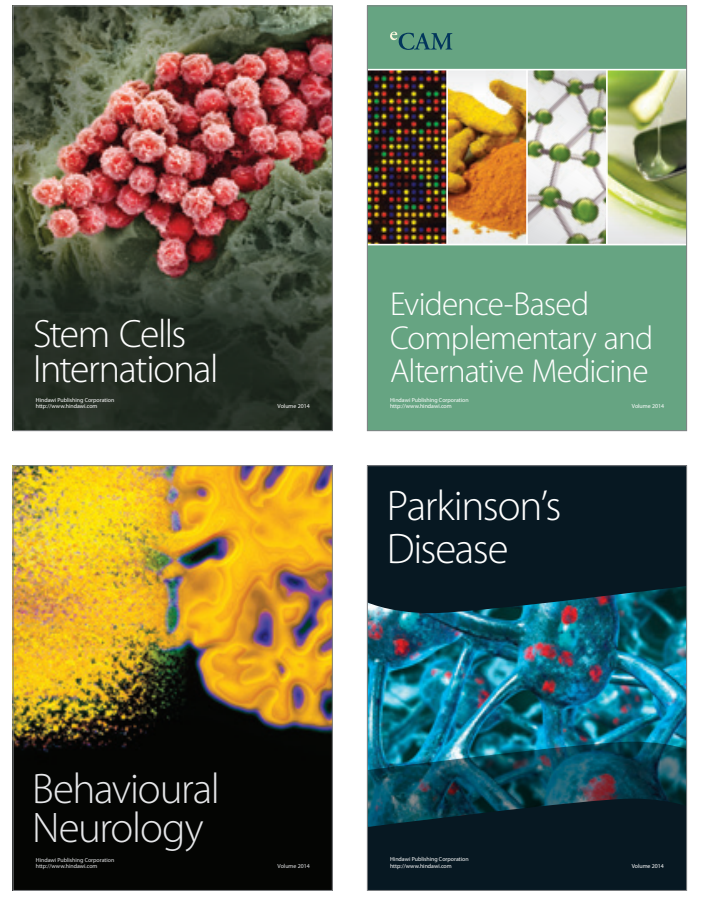
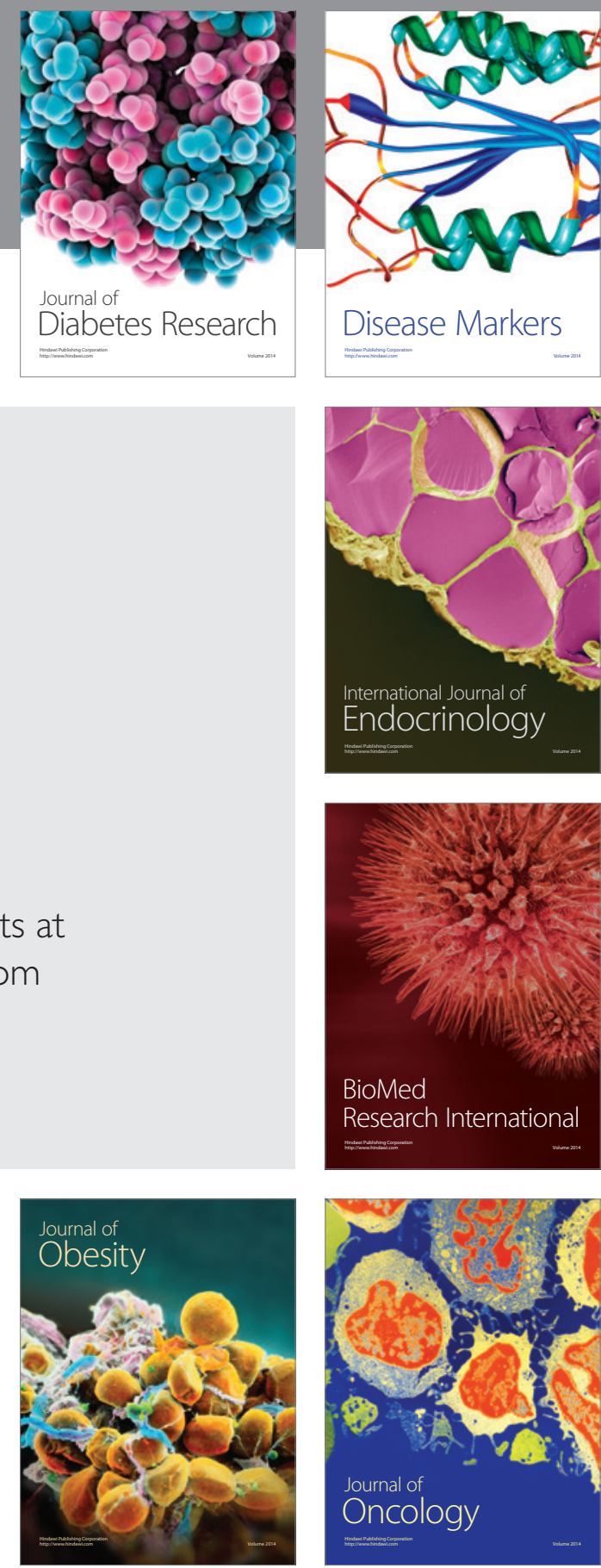

Disease Markers
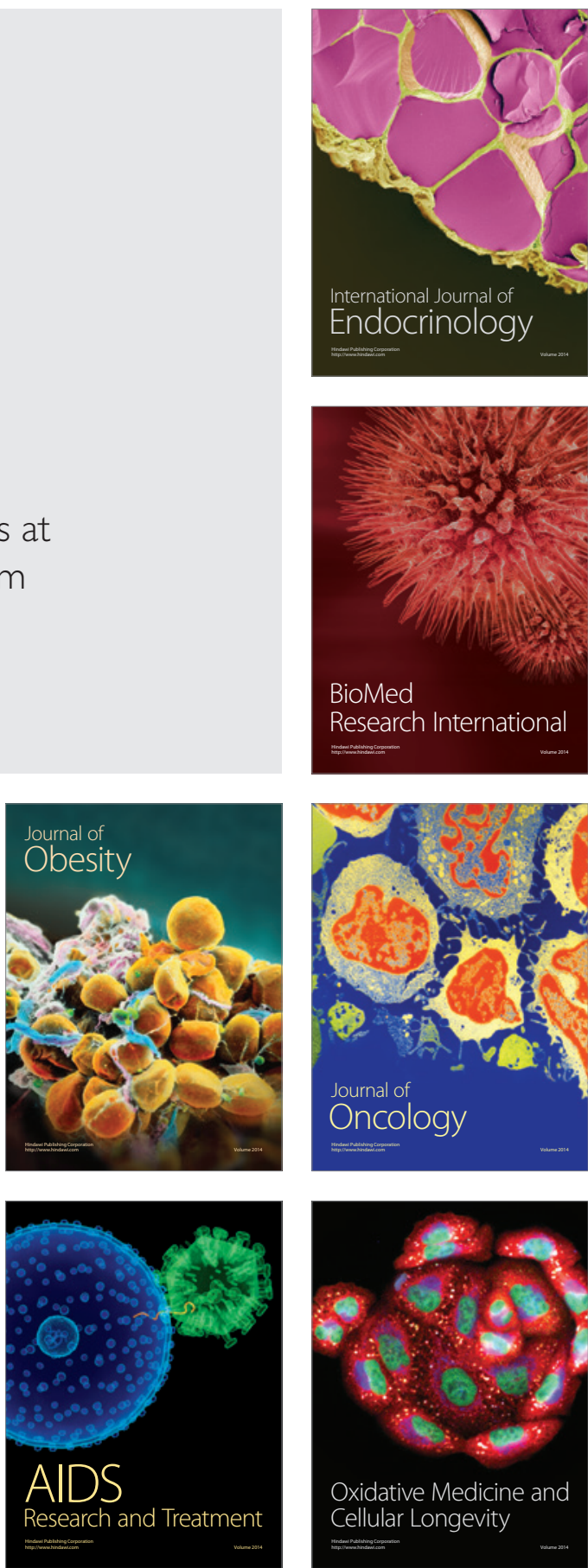